\title{
Operadores genéticos y de búsqueda aleatoria aplicados a tareas de selección de materiales de revestimiento para cucharas metalúrgicas
}

\author{
Osmel Martínez Valdés ${ }^{\mathrm{a}, \bigotimes}$, José Arzola Ruiz ${ }^{\mathrm{b}}$ \\ ${ }^{a}$ Grupo de Software, ACINOX Ingeniería, MINDUS, Calle 20 No 10522, 100-101, Cotorro, La Habana, Cuba \\ ${ }^{b}$ Centro de Estudios de Matemática para las Ciencias Técnicas, Universidad Tecnológica de La Habana "José Antonio Echeverría" \\ (CUJAE), Calle 114 NN$^{\circ} 11901,119-127$, Marianao, La Habana, Cuba \\ Autor para la correspondencia: osmelmv@gmail.com
}

Submitted: 18 Febrero 2016; Accepted: 24 Marzo 2017; Available On-line: 26 Julio 2017

\begin{abstract}
RESUMEN: La selección del revestimiento refractario de las cucharas se ha basado, hasta el presente, en criterios prácticos de los tecnólogos metalúrgicos. En el presente artículo se expone, por primera vez en la literatura especializada, la aplicación comparada de dos operadores de generación de opciones de materiales por zonas de acuerdo al esquema de descomposición en los algoritmos evolutivos multiobjetivos para el tratamiento de la tarea de selección de materiales. Se realizan las validaciones numéricas de funcionamiento de ambos operadores, así como, la comparación de sus resultados en cuanto a eficiencia y calidad de las soluciones obtenidas en las ejecuciones computacionales de ambos algoritmos. Estos operadores se implementaron bajo el concepto del método de Integración de Variables. En particular, se utiliza el operador de búsqueda aleatoria de un código variable y un operador genético de ordenamiento de soluciones no dominadas, basado en un algoritmo genético elitista (NSGAII) aplicados al problema estudiado.
\end{abstract}

PALABRAS CLAVE: Algoritmos evolutivos multiobjetivo; Método de integración de variables; Revestimiento de cucharas metalúrgicas; Selección de materiales

Citar como/Citation: Martínez Valdés, O.; Arzola Ruiz, J. (2017) "Operadores genéticos y de búsqueda aleatoria aplicados a tareas de selección de materiales de revestimiento para cucharas metalúrgicas”. Rev. Metal. 53 (3): e099. http://dx.doi.org/10.3989/revmetalm.099

\begin{abstract}
Genetic and random search operators applied to materials selection task of lining for metallurgical ladles. The selection of refractory lining of the ladles for steel casting has been based, until the present, in the metallurgy technologists' practical criteria. In this article the compared application of two search operators of materials options selection by zones inspired in the Evolutionary Multiple Objectives Algorithms for the treatment of the task of materials selection according to the adopted decomposition outline, is firstly reflected in the specialized bibliography. The numerical validation of the behavior of different indicators of both operators, as well as the comparison of their gain, efficiency and obtained solutions quality in the computational executions of both algorithms is carried out. These operators were implemented under the concept of the Integration of Variables method. Particularly, the Random Search of a Variable Code operator and a Not-Dominated Sorting Genetic Operator, based on elitist genetic algorithm NSGAII applied to the studied task are used.
\end{abstract}

KEYWORDS: Integration of variables method; Materials selection; Metallurgical ladles refractory lining; Multiple objective evolutionary algorithms

ORCID: Osmel Martínez Valdés (http://orcid.org/0000-0001-8065-0828); José Arzola Ruiz (http://orcid. org/0000-0003-1128-2245)

Copyright: (C) 2017 CSIC. Este es un artículo de acceso abierto distribuido bajo los términos de la licencia Creative Commons Attribution (CC BY) España 3.0. 


\section{INTRODUCCIÓN}

Como práctica adoptada en la bibliografía especializada y en la experiencia ingenieril, para la selección de materiales de revestimiento para instalaciones térmicas, se recurre al conocimiento empírico obtenido por los investigadores y tecnólogos para la evaluación de nuevas opciones de revestimiento, lo cual limita la posibilidad de evaluar un número considerable de opciones para realizar una adecuada toma de decisiones. Esta situación es muy frecuente en los talleres de producción de acero que utilizan la tecnología de metalurgia secundaria, donde la continua sustitución de los materiales de revestimiento para las cucharas de vaciado del acero se realiza a un ritmo considerable, según los niveles productivos del taller. La elección de la secuencia de materiales y los espesores adecuados para este tipo de instalación, constituye una tarea de ingeniería de elevada complejidad, no solo por las rigurosas condiciones de trabajo a las que se someten los materiales, sino también por la necesidad de que la elección de estos asegure un comportamiento eficiente de la cuchara metalúrgica con el propio entorno productivo.

El tratamiento más extendido en la literatura para la evaluación de los revestimientos refractarios toma en consideración solamente la reducción de las pérdidas de calor. Así, Schacht y Maupin (2004) en Refractories Handbook, exponen un análisis de este criterio en uno de los capítulos de esta obra. Sukul y Balaramakrishna (2011) argumentan a favor de este criterio y exponen consideraciones prácticas de aplicación. Wei y Singh (1986) exponen los criterios de compatibilidad entre el revestimiento y la carcasa metálica de las cucharas para evitar la deformación a partir de la reducción de las presiones de interacción resultantes. Barbés, et al. (2007) destacan la importancia de los criterios de desgaste. Chawla y Gupta (2010) exponen consideraciones para la elección de materiales que trabajan en ambientes corrosivos a altas temperaturas y destacan los beneficios productivos del desgaste de la pared de trabajo.

Clark et al. (2002) resaltan la importancia de considerar el comportamiento de las tensiones térmicas. Prietl et al. (2006) consideran las relaciones entre las propiedades de diseño y el comportamiento termo mecánico de la estructura refractaria. Boisse et al. (2001) exponen criterios de selección basados en la resistencia de las mamposterías refractarias a las variaciones de la carga térmica cíclica. Zabolotsky (2011) expone la importancia de considerar la resistencia al choque térmico producido por el cambio brusco de temperatura en la superficie de la pared de trabajo y expone un procedimiento para su determinación. Chlup et al. (2006) exponen los criterios a considerar para un buen comportamiento a la fractura de los materiales cerámicos sometidos a cargas térmicas cíclicas.
En los trabajos anteriores se destaca la utilización priorizada de cada criterio en consideración. Se requiere, sin embargo, un tratamiento integral del problema, tomando en consideración todos sus aspectos. En la ingeniería moderna se emplean varios enfoques para dar tratamiento a este problema, destacándose los métodos de selección de materiales basados en la comparación de propiedades y requerimientos (Brifcani, 2012). Estos métodos basan su tratamiento en la elección de materiales según la asignación de un ranking al caracterizar las propiedades por índices preferenciales (Chatterjee y Chakraborty, 2012).

El método más conocido es el propuesto por Ashby en sus obras Materials: Engineering, Science, Processing and Design (Ashby et al., 2010) y Materials Selection in Mechanical Design (Ashby, 2011), conocido por Ashby free-search. Este método consiste en la generación de un ranking de materiales a partir de la determinación de sus índices de desempeño, obtenidos por la relación entre sus propiedades. La selección de materiales ha sido tratada, además, a través de los métodos de decisión multicriterio TOPSIS, VIKOR y ELECTRE para, de igual forma obtener un ranking de materiales. Los trabajos de (Shanian y Savadogo, 2006; Jahan et al., 2011; Marzouk, 2011) constituyen ejemplos de aplicación de estos métodos a tareas de selección de materiales, en los cuales se incorporan niveles de importancia y pesos a los índices de propiedades (Findik y Turan, 2012; Rai et al., 2013). Jahan et al. (2010) lleva a cabo una revisión de los métodos de selección de materiales donde se resumen otros procedimientos de selección de enfoques similares. Estos métodos, al ser de carácter general, evaden conceptos determinantes del diseño de las cucharas de vaciado de acero, lo cual dificulta la posibilidad de considerar la relación directa de las variables de diseño del revestimiento refractario con el entorno productivo del taller, además de no permitir el tratamiento integrado del comportamiento heterogéneo de las zonas de esta instalación en su funcionamiento.

La evolución del pensamiento ingenieril ha conducido a otras formas de búsqueda de alternativas de solución al problema de selección de materiales basados en la aplicación de procedimientos evolutivos. Sapuan (2011) propone el empleo de Knowledge-based Engineering (KBE) a partir del conocimiento almacenado en bases de datos que registran el comportamiento de los materiales frente a varias condiciones de trabajo. Bassir et al. (2008), proponen un procedimiento de selección de materiales para la construcción de estructuras mecánicas utilizando Algoritmos Genéticos (AG) con variables mixtas. Sakundarini et al. (2013) utilizan los Algoritmos Genéticos para la selección de materiales reciclables para la elaboración de componentes de automóviles. En Sistemas de Ingeniería (Arzola, 2000), se propone el empleo de indicadores técnicos 
y económicos para caracterizar la eficiencia de una pared refractaria y establece procedimientos basados en la Optimización No lineal y Discreta para el tratamiento del problema de selección de espesores bajo criterios múltiples de paredes refractarias y aislantes.

Martínez (2011) propone un modelo matemático para el tratamiento de la tarea de selección de revestimiento refractario para cucharas de vaciado de acero, donde se presenta una formulación matemática que permite el tratamiento por zonas y la conciliación final de las combinaciones de materiales y espesores que conforman un revestimiento. (Martínez y Arzola, 2016) exponen la aplicación del operador de búsqueda aleatoria de códigos variables y realizan un análisis de su ejecución numérica, mostrando resultados satisfactorios. Por estar este operador poco estudiado y extendido en la bibliografía especializada, se hace necesaria su comparación con otros operadores evolutivos más estudiados en tareas de complejidad semejante a la estudiada, como lo es el procedimiento denominado NSGAII (Non-dominated Sorting Genetic Algorithm-II).

Por estas razones, el principal objetivo del presente artículo consiste, precisamente, en la comparación numérica de los resultados obtenidos por ambos operadores, demostrando la validez del uso de los operadores propuestos. Una novedad de este artículo consiste en que se aplica un operador genético inspirado en el NSGAII a tareas de selección de materiales para revestimientos metalúrgicos, no habiendo sido referenciado anteriormente su uso en aplicaciones como la presentada en este artículo.

\section{MATERIALES Y MÉTODOS}

\subsection{Formulación de la tarea de selección de revestimientos para cucharas metalúrgicas}

La selección de materiales refractarios y aislantes para cucharas de vaciado de acero constituye el centro de numerosas investigaciones encaminadas a la reducción de los costos del proceso de elaboración del acero a partir de la disminución del consumo de materiales por la continua sustitución del revestimiento. Esta tarea se logra a partir de considerar en el diseño un adecuado comportamiento de las pérdidas térmicas, el peso, los costos, la compatibilidad del revestimiento con la carcasa metálica, la resistencia al estrés y choque térmico de las capas, entre otros indicadores de desempeño del revestimiento (ver Fig. 1).

La propia configuración geométrica de esta instalación y las características del metal líquido y la escoria, trae como resultado un desigual comportamiento, lo cual necesariamente implica que la elección de los materiales ha de realizarse según las zonas de trabajo de las cucharas indicadas en la Fig. 1. De esta forma, el problema de selección de materiales

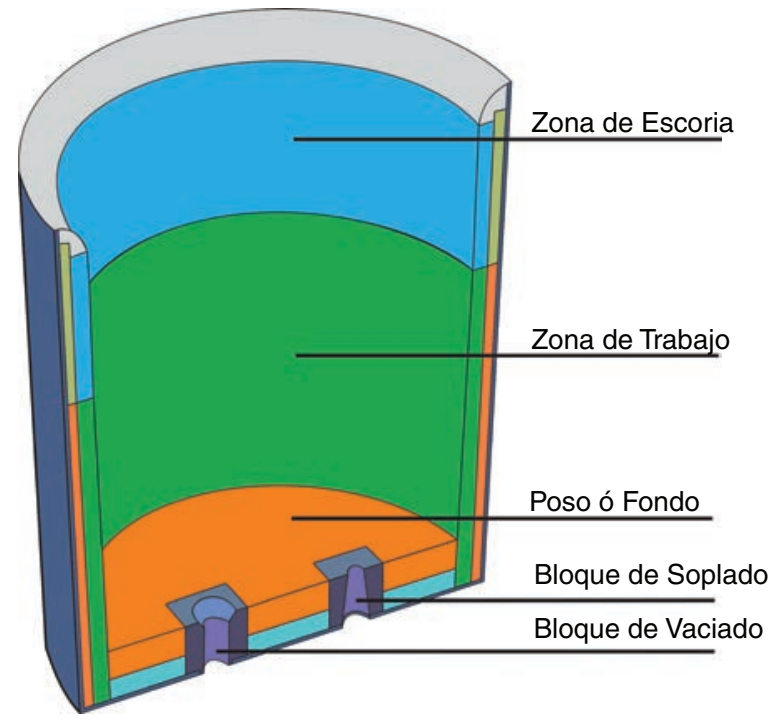

FIGURA 1. Representación de una cuchara de vaciado de acero, sus partes y zonas características (Martínez, 2011).

para las cucharas de vaciado de acero consiste, no solo en la selección de la secuencia de colocación de los materiales y sus espesores, sino que, además, ha de realizarse para cada zona, de forma que se asegure un comportamiento eficiente del revestimiento en su conjunto, lo que incrementa el nivel de complejidad del problema y dificulta la representación clásica del orden de complejidad $O\left(n^{k}\right)$ a partir del tamaño del problema $n$ y de su factor de crecimiento $k$. Una medida de la complejidad computacional para este problema puede formularse por la Ec. (1):

$$
I t=\prod_{i=1}^{c z}\left(m_{i} d_{i}\right)^{n c_{i}}
$$

donde, It es la cantidad de evaluaciones, $c z$ es la cantidad de zonas a considerar, $m_{i}$ la cantidad de materiales totales en la zona $i, d_{i}$ la cantidad máxima de posibles espesores de los materiales de la zona $i$ y $n c_{i}$ es la cantidad de capas de la zona. Se puede notar al evaluar la Ec. (1), que el número de evaluaciones crece de forma no polinomial al aumentar la cantidad de materiales, de capas y de zonas, lo que, sumado al carácter cualitativo de la variable material, obliga a su representación como código y convierte la tarea estudiada al tipo NP-hard (Arora y Barak, 2009). Esta complejidad se acentúa para cada una de las evaluaciones por la necesidad de utilizar procedimientos iterativos en el cálculo de los indicadores de desempeño y las restricciones de operación. Estas razones dificultan, además, el tratamiento directo del problema y justifican la utilización de enfoques heurísticos capaces de generar buenas soluciones en un tiempo razonable, con un empleo moderado de recursos computacionales. 
En la metodología de análisis sistémico expuesta por Arzola (2008), se propone el tratamiento de tareas complejas bajo estructuras de descomposición de diferentes tipos y las ventajas de realizar la descomposición de las tareas de ingeniería de forma tal que la estructura del modelo del proceso de toma de decisiones se haga corresponder con la del modelo matemático asociado, con la composición posterior de las opciones obtenidas. A partir de estos criterios, los autores plantean el modelo de optimización para la selección del revestimiento refractario de cucharas metalúrgicas y su descomposición según una estructura jerárquica participativa mediante el programa matemático siguiente (Martínez y Arzola, 2016):

$$
\begin{aligned}
& \underset{O_{R}}{\operatorname{Minimizar}}\left\{\max \left[w_{1}\left|\frac{C-C d}{C d}\right|, w_{2}\left|\frac{Q-Q d}{Q d}\right|, w_{3}\left|\frac{P-P d}{P d}\right|\right]\right\} \\
& \left(\delta b_{i}-\delta s_{i}\right)^{2} \leq \delta, i \in I \\
& B \geq B^{\min } \\
& h c \geq h^{\min } \\
& O_{R}=\left(O r_{1}, O r_{2}, \ldots, O r_{c z}\right)
\end{aligned}
$$

Donde, $O_{R}$ son las opciones generales de revestimiento de la cuchara, formadas a partir de los conjuntos de opciones de materiales y sus espesores $\left(O r_{1}, \mathrm{Or}_{2}, \ldots, O r_{c z}\right)$ generados por cada zona $i$ como resultado de la resolución de la tarea de optimización subordinada para la generación de opciones siguientes:

$$
\begin{aligned}
& \underset{O_{r i}}{\operatorname{Minimizar}}\left\{\max \left[w_{i}^{1}\left|\frac{c_{i}-c d_{i}}{c d_{i}}\right|, w_{i}^{2}\left|\frac{q_{i}-q d_{i}}{q d_{i}}\right|, w_{i}^{3}\left|\frac{p_{i}-p d_{i}}{p d_{i}}\right|\right]\right\} \\
& x_{i j}^{\min } \leq x_{i j} \leq x_{i j}^{\max }, \quad j=1, \ldots, n c_{i} ; i=1, \ldots, c z \\
& x_{i j} \in\left(d_{i j}^{1}, \ldots, d_{i j}^{k}\right), \quad j=1, \ldots, n c_{i} ; i=1, \ldots, c z \\
& T_{i j} \leq T_{i j}^{\max }\left(m_{i j}\right), \quad j=1, \ldots, n c_{i} ; i=1, \ldots, c z \\
& m i j \in M i, j \subset M i \subset M \\
& x i j \in X i j \subset X i \subset X
\end{aligned}
$$

$w_{1}, w_{2}$ y $w_{3}$ son los coeficientes que reflejan el peso o la importancia concedida a los indicadores de eficiencia respectivos relacionados mediante la Ec. (2), $w_{i}^{1}, w_{i}^{2}$ y $w_{i}^{3}$ constituyen de igual forma los pesos concedidos a los indicadores respectivos de la zona $i$ según la formulación (Ec. (7)). En ambos casos, los coeficientes de peso $w$ se normalizan mediante la restricción (Ec. (13)), donde $l$ es el número total de indicadores.

$$
\sum_{e=1}^{l} w_{e}=1, \quad w_{e} \in R, 0 \leq w_{e} \leq 1,
$$

$C$ es el costo total del revestimiento determinado por:

$C=\sum_{i=1}^{c z} \sum_{j=1}^{n c_{i}} C_{i j} x_{i j}$

Donde $c z$ es el número total de zonas, $n c_{i}$ es el número total de capas de materiales $m$ para la zona $i, C_{i j}$ es el costo por unidad de volumen del material en la zona $i$ de la capa $j$ y $x_{i j}$ es el espesor del material correspondiente, $C d$ es el costo total deseado del revestimiento. $Q$ son las pérdidas totales de calor determinadas mediante (Ec. (15)):

$Q=\sum_{i=1}^{c z} q_{i} A_{i}$

Donde, $q_{i}$ es la densidad de flujo calórico en la zona $i, A_{i}$ es el área de intercambio con el medio exterior correspondiente, $Q d$ son las pérdidas totales de calor deseadas a través de la carcasa de la cuchara. $P$ es el peso total del revestimiento determinado por (Ec. (16)):

$P=\sum_{i=1}^{c z} \sum_{j=1}^{n c_{i}} V_{i j} \rho_{i j}$

Donde, $V_{i j}$ es el volumen del material de la zona $i$ en la capa $j, \rho_{i j}$ es la densidad del material correspondiente, $P d$ es el peso deseado del revestimiento, $\delta b_{i}$ es la dilatación radial del revestimiento por zona, $\delta s_{i}$ es la dilatación radial de la chapa de la cuchara en la zona correspondiente, $I$ es el conjunto de zonas pertenecientes a las paredes laterales de la cuchara, $\delta$ es un valor permisible de diferencia de dilatación, $B$ es la cantidad de metal calculada en función del volumen interior de la cuchara según los espesores totales de las paredes de las zonas, $B^{\text {min }}$ es la cantidad mínima de metal a asegurar en la cuchara, $h c$ es la reserva de altura metalúrgica calculada en función del volumen de metal y escoria, $h^{\min }$ altura de reserva metalúrgica mínima requerida. $c_{i}, q_{i}$ y $p_{i}$ son el costo, la pérdida de calor y el peso totales por $\mathrm{m}^{2}$ de revestimiento respectivamente calculados para la zona $i . c d_{i}, q d_{i}$ y $p d_{i}$, corresponden al costo total deseado, la pérdida de calor total deseada y el peso total deseado por $\mathrm{m}^{2}$ de revestimiento respectivamente en la zona $i, x_{i j}{ }^{\text {min }}$ es el espesor mínimo establecido de la capa $j$ en la 
zona $i, x_{i j}$ es el espesor, $x_{i j}^{\max }$ el espesor máximo correspondiente, $d_{i j}{ }^{k}$ los valor discretos de los espesores desde uno hasta el máximo de combinaciones $k$ para cada capa $j$ en las zonas, $T_{i j}$ la temperatura interna de la capa $i, T_{i j}{ }^{\max }$ representa los valores máximos de trabajo de los materiales $m_{i j}$ respectivos, $M_{i j}$ es el conjunto de materiales indicados para una capa $j$ en la zona $i$ según una distribución predefinida y $X_{i j}$ es el conjunto de espesores permitidos para una capa $j$ en una zona $i$ según se una distribución determinada por los posibles espesores y sus combinaciones hasta un espesor máximo según los materiales escogidos para la capa.

Las relaciones (2) y (7) modelan el propósito de minimizar la distancia de Chebyshov ponderada y normalizada de los indicadores de eficiencia con respecto a valores deseados, los que se corresponden con los niveles de aspiración de Wierzbicki y se utiliza durante la generación y selección de las soluciones (Lewandowski y Wierzbicki, 1990; Jiménez, 2009). La determinación de las alturas de las zonas de la cuchara de vaciado se realiza como parte del cálculo de la función objetivo de la tarea de mayor nivel expresada en (2) y como parte del cálculo de la cantidad de metal a asegurar en la cuchara, penalizada por las restricciones (4) y (5) para una condición de diseño dada. Los objetivos de la expresión (7) se corresponden con los de la función original (2) para un $\mathrm{m}^{2}$ de pared y permiten seleccionar los materiales y espesores que minimizan la relación de compromiso entre los indicadores de la tarea de conciliación (2)-(6) en correspondencia con el sistema de preferencia del decisor. Para la determinación de los espesores óptimos de cada combinación de material a evaluar se utilizan los métodos de exploración de la programación no lineal y de ramificaciones y acotaciones de la programación discreta con el mismo tratamiento expuesto en (Arzola, 2000). Los valores deseados de cada uno de los indicadores, tanto por cada zona como para la tarea general, son establecidos a priori por el actor de la decisión a partir del conocimiento y experiencias prácticas.

A pesar de utilizar estos criterios de optimización, la descomposición realizada permite el empleo de otros criterios en sustitución de la función (7). El éxito del proceso propuesto en este artículo depende de una adecuada selección de materiales y sus espesores en las subetapas de optimización, donde se concentra el mayor peso de procesamiento $\mathrm{y}$, por tanto, el mayor consumo computacional. Esta selección requiere de la elaboración de procedimientos eficientes para la solución del problema combinatorio que se presenta y que requiere de la utilización de procedimientos de codificación de las variables que representen a los materiales y sus propiedades. Esta capacidad de tratamiento es propia de métodos que operen con variables integradas en códigos de varios tipos, como lo hace el método de Integración de Variables.

\subsection{El método de integración de variables}

El concepto se vincula a la representación compacta de opciones de solución de una tarea de optimización en códigos variables y a su evolución mediante operadores. Aunque este concepto se propone en (Arzola et al., 2003), la idea ha sido aplicada en otros trabajos. A partir del concepto de Integración de Variables, se han desarrollado nuevos algoritmos como los de Búsqueda y Exploración Aleatorias de Códigos Variables (Arzola, 2008), el de Búsqueda Aleatoria Condicionada (Marrero et al., 2015), y un procedimiento para la solución del problema del máximo clique ponderado (Toranzo y Arzola, 2014), los cuales utilizan números reales enteros como códigos de representación compacta de las soluciones. La codificación más extendida en la literatura para la representación de soluciones es la binaria, la cual es utilizada ampliamente en los Algoritmos Evolutivos Multiobjetivos (AEMO) (Coello et al., 2007). Entre las estrategias inspiradas en los procesos de evolución de las especies, destacan las que mantienen elitismo durante la búsqueda de soluciones. Ejemplos de AEMO elitistas son SPEA (Zitzler y Thiele, 1999), PAES (Knowles y Corne, 2000) y NSGAII (Kollat y Reed, 2005), siendo estos últimos reconocidos como los más eficientes en tareas de optimización basados en el ordenamiento de soluciones no dominadas (Srinivas y Deb, 1994). La amplia posibilidad y validez demostrada, motivaron su implementación para el tratamiento de la tarea de optimización multiobjetivo de selección de materiales correspondiente a $n$ zonas de una cuchara. La descripción de la implementación de los algoritmos desarrollados como operadores para la codificación y evolución de soluciones se exponen a continuación.

\subsubsection{Operador genético inspirado en el ordenamiento de soluciones no dominadas}

El objetivo fundamental para considerar como referencia este tipo de algoritmo, es su capacidad de tener siempre disponible a los individuos más aptos para la reproducción a través de su enfoque elitista. El elitismo es implementado utilizando una población externa, en la cual, se almacenan las soluciones no dominadas encontradas (Deb et al., 2002). Basado en esta idea el algoritmo implementado para la selección de materiales en las subtareas de optimización se muestra en la Fig. 2, el cual se inicia con la creación de la población inicial PopulationElit. Esta población es creada a partir de la selección aleatoria de opciones de solución codificadas compuestas por dígitos binarios.

Codificación: Para la representación de las soluciones se utilizó la codificación basada en cadenas de dígitos binarios cuya longitud máxima se determina por la cantidad máxima de posibles combinaciones 


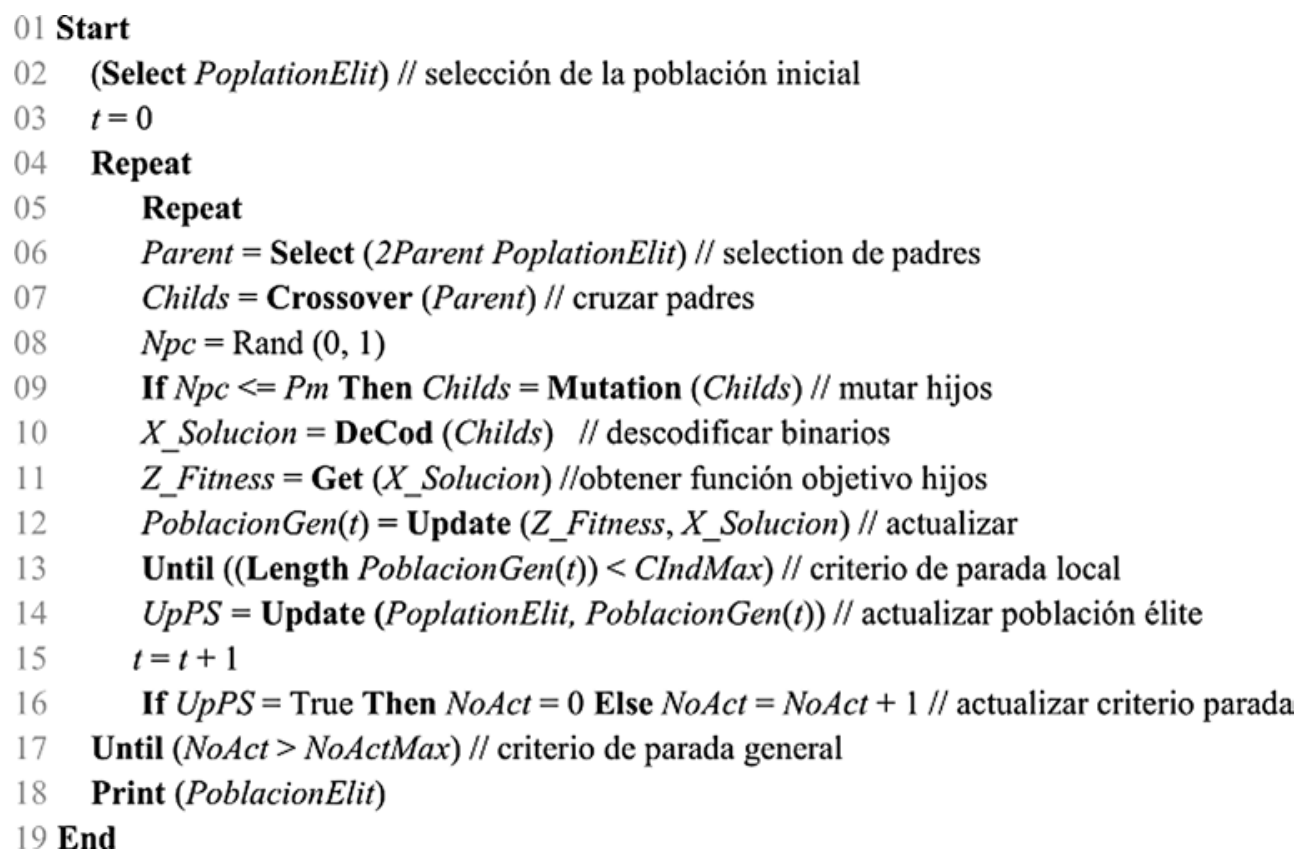

FIgURA 2. Pseudocódigo del operador genético inspirado en el NSGAII.

de materiales Inter dado por la expresión (17), donde $m$ es el número de materiales totales disponibles para cada capa $j$ hasta el número máximo de capas $n c_{i}$ de la zona $i$.

Inter $=\left[\prod_{j=1}^{n c_{i}} m_{j}\right]-1$

Decodificación: Cada cadena binaria generada representa una secuencia de números enteros correspondientes a los índices que identifican a los materiales a evaluar. Por ejemplo, si se tiene la cadena

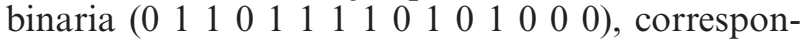
diente a una opción de revestimiento de tres capas, el material de cada capa estaría representado por el número entero de cada subcadena obtenida al dividir la cadena en tres $\left\{\begin{array}{lllll}0 & 1 & 1 & 0 & 1\end{array}\right),\left(\begin{array}{lllll}1 & 1 & 1 & 0 & 1\end{array}\right)$ y $\left(\begin{array}{llll}0 & 1 & 0\end{array}\right.$ $00)\}$. En este ejemplo general, el revestimiento estaría conformado por los materiales representados por el índice 13, 29 y 8, según el orden establecido a priori. Creada esta población inicial, a partir de la codificación-decodificación expuesta, el algoritmo continúa con un doble ciclo de iteraciones. El ciclo más interno se encarga de la generación de nuevos individuos o soluciones a partir de los mecanismos de selección, cruce y mutación según la estrategia evolutiva seleccionada para el caso en estudio.

Estrategias de evolución de códigos: La estrategia está dada por una selección binaria por torneo, para ello se determina a cada individuo de PopulationElit su función de aptitud según (Ec. (7)) y son ordenados para realizar la selección del individuo más apto de la población como primer padre. El segundo

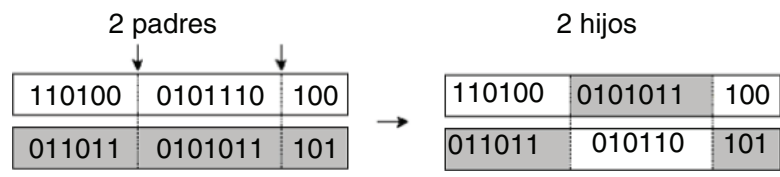

FIgURA 3. Representación de la reproducción por dos puntos de cortes.

padre se selecciona de forma aleatoria del resto de las soluciones ordenadas sin aplicar probabilidades, para posibilitar el aumento de la diversidad genética de las soluciones hijas. Una vez realizada la selección de los padres, se realiza una reproducción por el método de cruce de dos puntos el cual consiste en seleccionar aleatoriamente dos posiciones en las cadenas binarias y combinar los segmentos resultantes según se muestra en la Fig. 3. Una vez obtenidos los nuevos hijos, se aplica un operador de mutación basada en orden (swap mutation) de dos puntos con una probabilidad de 0,01 , el que consiste en la selección de dos genes aleatorios y su intercambio de posiciones. Esta mutación se aplica si, al generar un número aleatorio $N p c$ entre cero y uno, su valor es menor o igual a la probabilidad establecida. Tanto el mecanismo de selección como el de mutación, tienen como objetivo acentuar la posibilidad de generar soluciones hijas con nuevas características basadas en el genotipo de las mejores soluciones, lo que implica, además, ampliar el radio de búsqueda y detectar soluciones no exploradas para fomentar una mayor diversidad de soluciones finales (Méndez, 2008).

Procedimientos para la creación y actualización de las poblaciones: Aplicada la estrategia evolutiva, se 
realiza la decodificación de las soluciones hijas por la función DeCod y se calculan los valores de función objetivo $Z$ _Fitness. La función (7) se utiliza con el objetivo de determinar la cercanía de cada solución generada a una deseada utilizando la distancia de Chebyshov, lo que permite hallar y ordenar las soluciones próximas al frente de Pareto y, por tanto, soluciones próximas a las no dominadas encontradas por el algoritmo, y se utilizan inicialmente para la creación de la población PoblacionGen(t) (Méndez, 2008). Este orden cíclico se repite hasta que se complete la población con una cantidad de individuos igual a CIndMax, lo que constituye el criterio de parada de la creación de la nueva generación de individuos. Una vez alcanzado el número máximo de individuos, se procede a realizar la actualización de la población élite del algoritmo, donde a partir del ordenamiento por el valor de su función objetivo se incluyen los individuos más aptos, o sea, las soluciones no dominadas encontradas. La actualización de la población élite una vez completada se realiza a partir de la eliminación de aquellas soluciones con peores valores de $Z$ _Fitness. Este mecanismo permite que continuamente estén disponibles las mejores soluciones para que los padres de las nuevas generaciones de códigos puedan ser elegidos de esta población élite por el operador selección. A medida que el algoritmo continúa explorando, se van generando soluciones que no actualizan la población élite, lo que se refleja en la variable $(U p P S=$ False $)$, ya sea por ser ineficientes o por estar ya incluidas en las poblaciones predecesoras. Luego de transcurrido un número de veces sin que se actualice esta población (NoAct > NoActMax) el algoritmo da por terminada la búsqueda de soluciones de la tarea.

\subsubsection{Operador de Búsqueda Aleatoria de Códigos Variables}

A diferencia del operador genético, el algoritmo desarrollado para la aplicación del operador de búsqueda aleatoria de códigos variables está compuesto por un único ciclo de búsqueda dirigido a la exploración de una secuencia ordenada de códigos numéricos reales enteros entre 0 e Inter. En este algoritmo, la localización de las soluciones óptimas se realiza a partir de la discriminación de los intervalos de búsqueda, obtenidos al seleccionar dos soluciones aleatorias en la secuencia ordenada de códigos (Martínez y Arzola, 2016). De igual forma que en el operador anterior, se emplea la función (Ec. (7)) para evaluar las distancia entre las soluciones generadas con la solución ideal o deseada. Otra diferencia a resaltar es que, en este operador, la población se actualiza directamente a partir de las soluciones generadas durante la búsqueda. Para garantizar la exploración de nuevas zonas de la serie de códigos compactos, los límites se restauran a sus valores iniciales (0-Inter) y se repite el procedimiento de cortes aleatorios hasta obtener un número de iteraciones seguidas NoActMax sin que se actualice la población.

\subsubsection{Conciliación de soluciones de revestimiento de cucharas}

La aplicación de los operadores expuestos a cada una de las zonas consideradas, dan como resultado un número de opciones de secuencias de materiales y espesores optimizadas por cada una de ellas. La tarea superior de conciliación selecciona, a partir de estas opciones de revestimiento, aquellas combinaciones que optimizan los criterios generales de selección de revestimientos. Como algoritmo de conciliación de opciones generales de revestimientos en el presente trabajo se utiliza el algoritmo de búsqueda del extremo de una función de código variable (Arzola, 2008), donde las opciones a codificar (Alternativas) son las soluciones de combinaciones de materiales y espesores obtenidas para cada zona, y como función objetivo se evalúa la expresión (2) con las restricciones (3)-(6). Los procedimientos para la decodificación, búsqueda, creación y actualización de la población final de opciones de revestimiento se ajustan exactamente a lo descrito por los autores en (Martínez y Arzola, 2016).

\section{RESULTADOS}

\subsection{Análisis de resultados de la aplicación de los operadores de búsqueda de solución}

El análisis de eficacia de la aplicación de los operadores descritos se centra en la descripción de los comportamientos computacionales correspondientes a partir del análisis de sus desempeños y la calidad relativa de las soluciones finales de ambos. La bibliografía especializada expone varios tratamientos para evaluar el rendimiento de procedimientos heurísticos implementados en ordenadores basados fundamentalmente en la medición del tiempo real de ejecución del algoritmo para así caracterizar con solidez su desempeño (Wegener, 2005; Hromkovič, 2011). Para ello, se realizó la implementación en ordenador de los operadores descritos de acuerdo a lo descrito en (Martínez y Arzola, 2016) para posibilitar igualdad de condiciones de ejecución. Se utilizó el lenguaje AutoLISP en la plataforma de diseño gráfico AutoCAD 2014 con licencia educativa, el cual está adaptado al trabajo con inteligencia artificial y facilita la programación de los procedimientos referidos en este trabajo y, además, permite la representación gráfica de soluciones propias de los sistemas CAD, para la su implementación en la práctica ingenieril. Para la gestión de los datos se creó un fichero con una estructura específica interpretado por el propio sistema y adaptado a las características funcionales del lenguaje de programación. Para la ejecución 
de los cálculos numéricos se empleó un ordenador de trabajo, modelo Toshiba Satellite L870, con un microprocesador Intel I5-3230 a 2,6 GHz de doble núcleo y memoria RAM de 4GB a 1,600 MHz con sistema operativo Windows 7 Ultimate.

Se seleccionaron los criterios de configuración de cucharas predominantes en la bibliografía y en la práctica de talleres de producción de acero por metalurgia secundaria (Prietl et al., 2006). Se establecieron 3 zonas con 3 capas posibles de materiales a seleccionar. Como cuchara de referencia se utilizó un modelo estándar de paredes rectas y fondo plano redondeado con $3,315 \mathrm{~m}$ de altura y $2,840 \mathrm{~m}$ de diámetro para la evacuación de 70 toneladas de acero líquido a $1650^{\circ} \mathrm{C}$. Se creó una base de datos con los materiales refractarios recomendados por el catálogo de materiales de la firma DIDIER con un total de 183 opciones de materiales posibles para cada capa (DIDIER, 1996).

\subsubsection{Análisis de resultados computacionales}

Se realizaron 100 ejecuciones de cálculo numérico por cada uno de los algoritmos de forma independiente con 2460374 opciones posibles de combinaciones de materiales por cada zona para obtener un total de 20 soluciones en la población de opciones de materiales y espesores finales. En todos los casos se mantuvieron fijos los pesos de los indicadores de las funciones objetivos e iguales a 0,33 . Con estas poblaciones de opciones se obtuvieron un total de 7999 opciones de revestimiento, las cuales fueron evaluadas por la tarea de conciliación para la generación de la población final con un tamaño de 20 individuos. Para estas ejecuciones se registraron los valores medios y los principales estadígrafos de los siguientes indicadores:

- CI, cantidad total de cálculos de la función de objetivo realizados para alcanzar la población final.

- $\quad T C$, tiempo computacional empleado para la determinación de la población de soluciones en milisegundos.
- $\quad V F O$, valor mínimo alcanzado por la función objetivo (mejor solución).

- $\quad V M F$, valor promedio de la función objetivo alcanzado por la población de soluciones.

- $D C$, diversidad de códigos de la población obtenida como la suma de las desviaciones absolutas normalizadas entre los códigos de solución generados con respecto a su valor medio.

Para cada indicador se hallaron los valores promedios $V M$, las desviaciones típicas $D T$, los coeficientes de asimetría típica $C A$ y curtosis tipificada $C K$ propias de las distribuciones para cada indicador. Los coeficientes de asimetría y curtosis estandarizados son de particular interés para determinar si la muestra procede de una distribución normal.

\subsubsection{Resultados obtenidos con el operador genético $(O G)$}

En las Tablas 1 y 2 se dan los resultados de las mediciones del desempeño del algoritmo del Operador Genético de Ordenamiento de Soluciones No Dominadas. Estos estadígrafos describen las medidas de posición, variabilidad y de forma del comportamiento de la muestra experimental obtenida.

La cantidad de iteraciones medias totales realizadas en la búsqueda de soluciones es de 35313,84 , pequeña en comparación con la cantidad de individuos posibles a explorar. El tiempo computacional total medio de ejecución es aproximadamente de $93200,85 \mathrm{~ms}$, con un tiempo promedio por iteración de $2,639 \mathrm{~ms}$, siendo el tiempo total de búsqueda de $6498204,986 \mathrm{~ms}$, para un 1,43\% del tiempo real de ejecución con respecto a la búsqueda exhaustiva. El valor de la función objetivo de la mejor solución es pequeño mientras que el valor promedio para el caso de las zonas es relativamente lejano con relación a su mejor. La diversidad de códigos de las poblaciones obtenidas oscila entre 0,44 y 0,52 lo cual representa cerca de un $47 \%$ de variabilidad de soluciones.

TABLA 1. Resultados computacionales de la experimentación por zona para el operador genético ${ }^{\mathrm{a}}$

\begin{tabular}{|c|c|c|c|c|c|c|c|c|c|c|c|c|}
\hline \multirow[b]{2}{*}{ Indicador } & \multicolumn{4}{|c|}{ Zona de Escoria } & \multicolumn{4}{|c|}{ Zona de Trabajo } & \multicolumn{4}{|c|}{ Piso } \\
\hline & VM & DT & $\mathbf{C A}$ & CK & VM & DT & $\mathbf{C A}$ & CK & VM & DT & $\mathbf{C A}$ & CK \\
\hline $\mathrm{CI}$ & 11265,38 & 189,238 & 0,537 & $-0,747$ & 11286,67 & 179,568 & 0,320 & $-0,903$ & 11275,4 & 183,699 & 0,112 & $-1,021$ \\
\hline $\mathrm{TC}$ & 17955,81 & 1217,09 & 1,150 & 1,106 & 16876,21 & 7545,97 & 1,147 & 1,999 & 19258,1 & 8836,64 & 1,714 & 4,966 \\
\hline VFO & 0,046 & 0,008 & 0,302 & $-0,842$ & 0,046 & 0,003 & $-0,053$ & $-2,081$ & 0,043 & 0,002 & 0,174 & $-1,924$ \\
\hline VMF & 1,144 & 0,032 & 2,003 & 1,946 & 1,092 & 0,085 & 1,358 & 1,926 & 1,747 & 0,059 & 2,002 & 1,061 \\
\hline $\mathrm{DC}$ & 0,447 & 0,121 & $-0,458$ & $-0,151$ & 0,462 & 0,141 & $-0,806$ & 0,984 & 0,445 & 0,124 & $-0,575$ & 0,053 \\
\hline
\end{tabular}

${ }^{a} V M$-valores promedios, $D T$ - desviaciones típicas, $C A$ - coeficientes de asimetría típica, $C K$ - curtosis tipificada, $C I$ - cantidad total de cálculos de la función de objetivo, $T C$ - tiempo computacional, $V F O$ - valor mínimo de función objetivo (mejor solución), $V M F$ - valor promedio de la función objetivo y $D C$ - diversidad de códigos de la población obtenida. 


\subsubsection{Resultados obtenidos con el operador de búsqueda aleatoria (OBA)}

En las Tablas 3 y 4 se muestra el resumen de los resultados computacionales para las zonas y para la tarea de conciliación. La cantidad de iteraciones medias totales realizadas en la búsqueda de soluciones es de 34736,42. El tiempo computacional total medio de ejecución es aproximadamente de $77855,73 \mathrm{~ms}$, con un tiempo promedio por iteración de 2,241 ms, siendo el tiempo total de búsqueda de $5513698,13 \mathrm{~ms}$, para un 1,41\% del tiempo real de ejecución con respecto a la búsqueda exhaustiva.

Los valores de las funciones objetivos son pequeños y próximos entre sí, lo cual muestra la obtención de poblaciones estables. La diversidad de códigos de las poblaciones obtenidas oscila entre 0,46 y 0,52 lo que se interpreta en aproximadamente un $49 \%$ de variabilidad en la composición de las soluciones de las poblaciones.

\section{DISCUSIÓN}

Para la realización de la comparación entre ambos operadores, se realizó un resumen de los promedios de los indicadores obtenidos de la experimentación

TABLA 2. Resumen de resultados computacionales para la tarea de conciliación ${ }^{b}$

\begin{tabular}{lcccc}
\hline & \multicolumn{4}{c}{ Tarea de conciliación general } \\
\cline { 2 - 5 } Indicador & VM & DT & CA & CK \\
\hline CI & 1486,33 & 253,45 & 0,174 & $-0,322$ \\
TC & 39110,65 & 16392,82 & 2,004 & 1,102 \\
VFO & 0,347 & 0,036 & 1,325 & 0,341 \\
VMF & 0,380 & 0,063 & 0,583 & $-1,244$ \\
DC & 0,523 & 0,232 & 0,682 & 0,267 \\
\hline
\end{tabular}

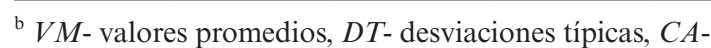
coeficientes de asimetría típica, $C K$ - curtosis tipificada, $C I$ cantidad total de cálculos de la función de objetivo, $T C$ - tiempo computacional, $V F O$ - valor mínimo de función objetivo (mejor solución), $V M F$ - valor promedio de la función objetivo y $D C$ diversidad de códigos de la población obtenida. para la selección de materiales por zonas, la tarea conciliadora y las iteraciones y el tiempo total de ejecución general. Los resultados de este resumen se muestran en la Tabla 5 del presente artículo.

Como se puede apreciar en la Tabla 5, para el caso de la selección de materiales, el OBA presenta un mejor comportamiento en general el cual realiza 499,21 iteraciones menos que el OG con un ahorro de tiempo de $15312,5 \mathrm{~ms}$, obteniéndose una población de soluciones con mejores valores de función objetivo ( $V F O p$ ) mayor diversidad de individuos $(D C p)$, a pesar que el OG logró mejores valores medios de función objetivo $(V M F p)$ en las poblaciones de opciones de materiales. También se puede observar que el desempeño en general del OBA es mejor con una eficiencia de un $28 \%$ superior al $O G$, aunque en la realidad ambos operadores obtienen muy buenos resultados, lo que se demuestra por la cercanía de los valores de los indicadores de calidad de las poblaciones de opciones de materiales obtenidos.

Para el caso de la tarea de conciliación de soluciones finales, el empleo de los OBA y OG presentan comportamientos muy cercanos, tanto en número de iteraciones como en tiempo computacional con una diferencia de apenas 78,21 iteraciones y 32,38 ms.

TABLA 4. Resumen de resultados computacionales para la tarea de conciliación general ${ }^{\mathrm{d}}$

\begin{tabular}{lcccc}
\hline & \multicolumn{4}{c}{ Tarea de conciliación general } \\
\cline { 2 - 5 } Indicador & $\mathbf{V M}$ & $\mathbf{D T}$ & $\mathbf{C A}$ & $\mathbf{C K}$ \\
\hline CI & 1408,12 & 284,01 & 0,176 & $-0,293$ \\
TC & 39077,95 & 8707,49 & 0,966 & 0,448 \\
VFO & 0,390 & 0,069 & 1,677 & 1,404 \\
VMF & 0,443 & 0,094 & 0,902 & $-0,842$ \\
DC & 0,510 & 0,328 & 0,357 & $-0,215$ \\
\hline
\end{tabular}

${ }^{\mathrm{d}} V M$ - valores promedios, $D T$ - desviaciones típicas, $C A$ coeficientes de asimetría típica, $C K$ - curtosis tipificada, $C I$ cantidad total de cálculos de la función de objetivo, $T C$ - tiempo computacional, $V F O$ - valor mínimo de función objetivo (mejor solución), $V M F$ - valor promedio de la función objetivo y $D C$ diversidad de códigos de la población obtenida.

TABLA 3. Resultados computacionales de la experimentación por zona para el operador de búsqueda aleatoria ${ }^{\mathrm{c}}$

\begin{tabular}{|c|c|c|c|c|c|c|c|c|c|c|c|c|}
\hline \multirow[b]{2}{*}{ Indicador } & \multicolumn{4}{|c|}{ Zona de Escoria } & \multicolumn{4}{|c|}{ Zona de Trabajo } & \multicolumn{4}{|c|}{ Piso } \\
\hline & VM & DT & $\mathbf{C A}$ & CK & VM & DT & $\mathbf{C A}$ & CK & VM & DT & CA & CK \\
\hline $\mathrm{CI}$ & 11604,02 & 129,64 & 0,522 & $-0,157$ & 11019,61 & 151,16 & 0,613 & 0,219 & 10704,67 & 163,09 & 0,272 & $-1,027$ \\
\hline $\mathrm{TC}$ & 12617,98 & 6599,46 & 2,079 & 1,269 & 13051,35 & 4777,29 & 1,614 & 2,749 & 13108,45 & 4609,38 & 1,665 & 4,621 \\
\hline VFO & 0,037 & 0,002 & $-0,095$ & $-1,652$ & 0,039 & 0,003 & $-0,080$ & $-1,679$ & 0,035 & 0,071 & 0,179 & $-1,921$ \\
\hline VMF & 0,421 & 0,049 & 1,354 & 1,902 & 0,319 & 0,038 & 1,112 & 1,899 & 0,431 & 0,061 & 1,025 & 1,631 \\
\hline $\mathrm{DC}$ & 0,521 & 0,320 & $-0,321$ & 0,207 & 0,512 & 0,299 & $-0,382$ & 1,611 & 0,460 & 0,202 & $-0,339$ & $-0,349$ \\
\hline
\end{tabular}

${ }^{c} V M$ - valores promedios, $D T$ - desviaciones típicas, $C A$-coeficientes de asimetría típica, $C K$ - curtosis tipificada, $C I$ - cantidad total de cálculos de la función de objetivo, $T C$ - tiempo computacional, $V F O$ - valor mínimo de función objetivo (mejor solución), $V M F$ - valor promedio de la función objetivo y $D C$ - diversidad de códigos de la población obtenida. 
TABLA 5. Resumen de ejecución de los operadores implementados ${ }^{e}$

\begin{tabular}{|c|c|c|c|c|c|c|c|c|c|c|c|c|}
\hline \multirow[b]{2}{*}{ Operador } & \multicolumn{5}{|c|}{ Selección de materiales por zonas } & \multicolumn{5}{|c|}{ Tarea de conciliación } & \multicolumn{2}{|c|}{ Ejecución Total } \\
\hline & CI & TC & VFOp & VMFp & DCp & CI & TC & VFOp & VMFp & DCp & CI & TC \\
\hline OG & 33827,51 & 54090,2 & 0,045 & 1,327 & 0,451 & 1486,33 & 39110,65 & 0,347 & 0,380 & 0,523 & 35313,84 & 93200,85 \\
\hline OBA & 33328,30 & 38777,7 & 0,037 & 0,394 & 0,497 & 1408,12 & 39077,95 & 0,390 & 0,443 & 0,510 & 34736,42 & 77855,73 \\
\hline
\end{tabular}

e OG- Operador Genético, OBA- Operador de Búsqueda Aleatoria, CI- cantidad total de cálculos de la función de objetivo, $T C$ - tiempo computacional, $V F O p$ - valor promedio de mejor función objetivo, $V M F p$-valor promedio de función objetivo de la población y $D C p$ - diversidad de individuos promedio.

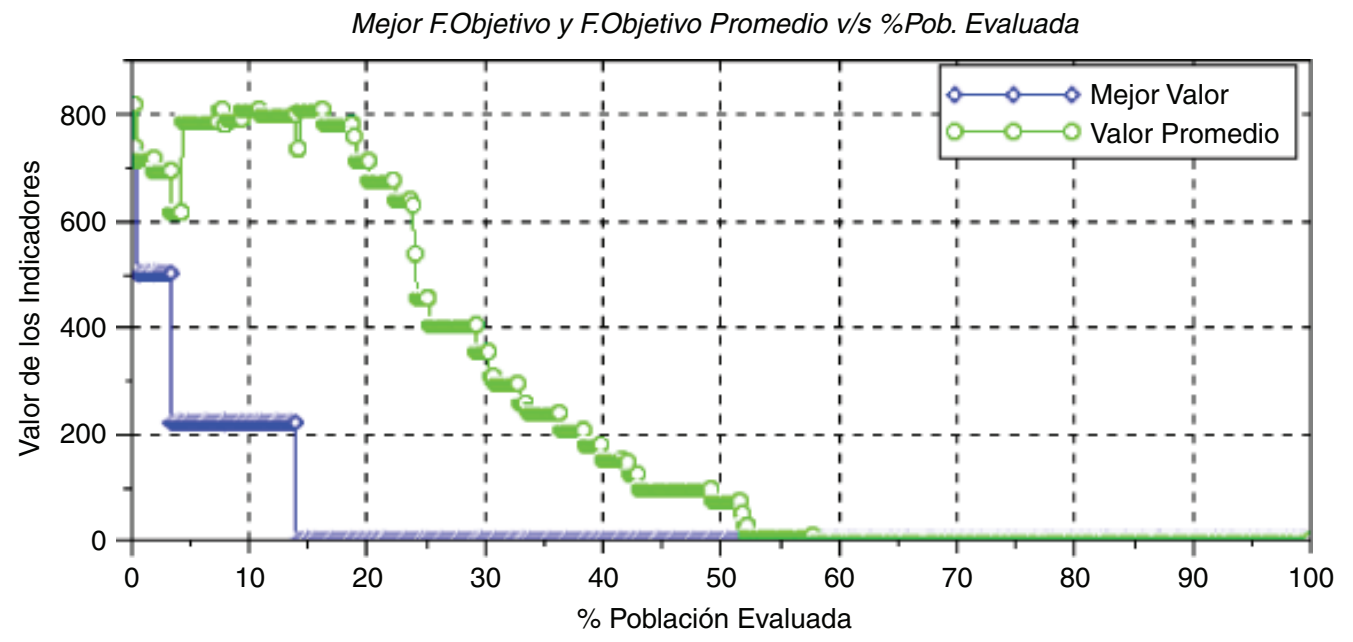

Figura 4. Comportamiento del mejor valor de la función objetivo y su promedio en la población evaluada para la zona de escoria con el operador genético implementado (OG).

El comportamiento de los restantes indicadores para la tarea de conciliación es muy similar, aunque ligeramente mejores en el caso del OG, mostrando una mejor diversidad de soluciones y mejores valores de función objetivo en la población final. En cuanto al desempeño general, el empleo del OBA presenta mejor rendimiento en cuanto a cantidad de iteraciones totales realizadas y tiempo computacional invertido en la solución de la tarea planteada en este artículo, con un ahorro total de 577,42 iteraciones y 15,345 segundos, lo que equivale a un $16,46 \%$ más eficiente que el OG. La tarea de selección de revestimientos requiere que los operadores implementados aseguren una convergencia adecuada, lo que implica que a medida que se realiza la búsqueda se deben obtener buenas soluciones que permitan mejorar las poblaciones de soluciones. Para analizar este comportamiento, se grafican los valores de la función objetivo de la mejor solución encontrada y de los valores promedios de la función objetivo de la población generada con respecto al porcentaje de individuos evaluados en las ejecuciones realizadas para cada operador. Los resultados de la evolución de las soluciones obtenidas por cada algoritmo se muestran en las Figs. 4 y 5.

En el caso del OG se observa que al inicio el valor promedio de la función objetivo de la población se mantiene elevado hasta cerca del $20 \%$ de las soluciones de la población. A partir de este valor comienza a descender por pequeños saltos. Esto se debe a que, en principio, el procedimiento de creación de la población al inicio considera todas las soluciones encontradas hasta completar la población élite y posteriormente la actualización se realiza en función de las subpoblaciones de descendientes generadas por los procedimientos de selección, cruce y mutación del algoritmo, lo cual justifica la mejora a saltos de los individuos de la población final. Igualmente, la función objetivo del individuo más apto va disminuyendo su valor a saltos un poco más distantes. Al trascurrir entre un $50 \%$ y un $60 \%$ de las evaluaciones, el valor promedio de la función objetivo tiende a valores pequeños, muy próximos al valor de la función objetivo de la mejor solución encontrada, lo cual indica que el procedimiento de búsqueda converge hacia valores pequeños de función objetivo, es decir hacia la búsqueda de mejores soluciones de materiales.

Para el caso del OBA mostrado en la Fig. 5, se observa un comportamiento menos escalonado, donde al inicio el valor promedio de la función objetivo de la población se mantiene elevado hasta que se evalúa cerca del 5\% de las soluciones. Esto se debe a que, en principio, el procedimiento de creación de 


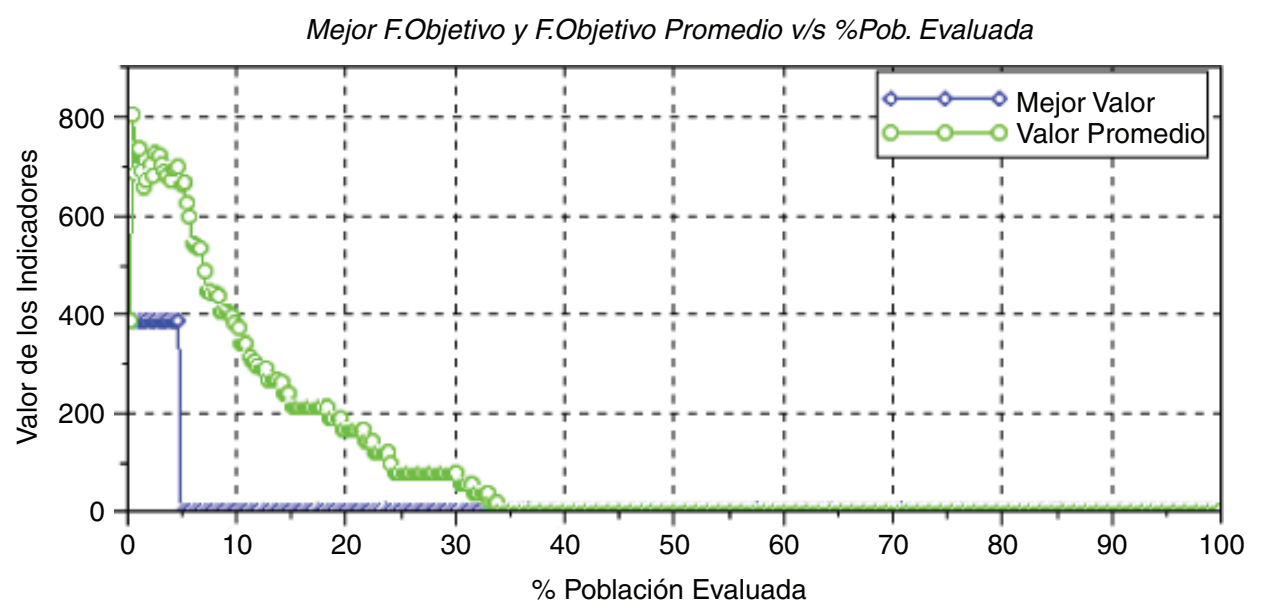

FIGURA 5. Comportamiento del mejor valor de la función objetivo y su promedio en la población evaluada para la zona de escoria con el operador de búsqueda aleatoria implementado (OBA).

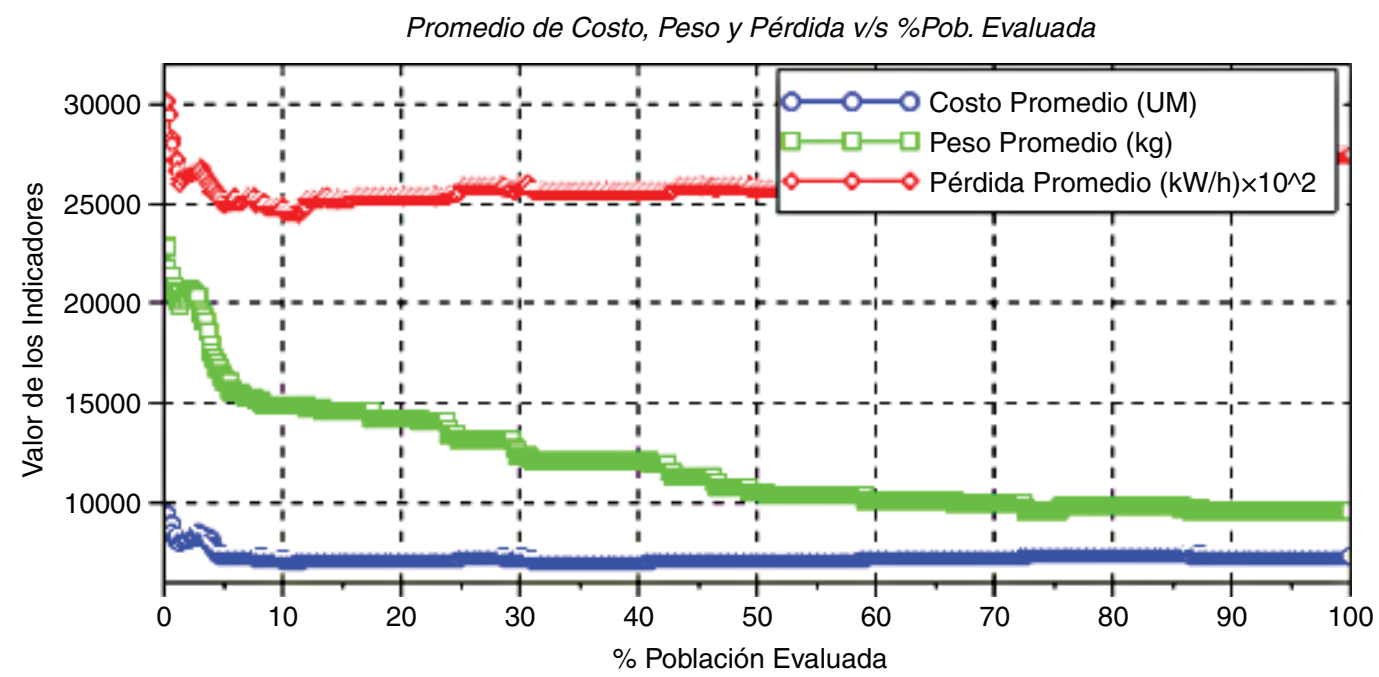

Figura 6. Comportamiento de los valores de los indicadores promedios de eficiencia de la población en la ejecución de la tarea conciliadora para el caso de aplicación del operador genético (OG).

la población al inicio considera todas las soluciones encontradas, por lo que no hay evolución, incluso pueden entrar en la población soluciones penalizadas alejadas de las eficientes. Una vez completada la población inicial, comienza inmediatamente la discriminación de aquellas soluciones consideradas como peores frente a las nuevas que se van encontrando en el proceso de búsqueda. Al trascurrir aproximadamente entre un $30 \%$ y un $40 \%$ de evaluaciones, el valor promedio de la función objetivo tiende a valores pequeños, muy próximos al valor de la función objetivo de la mejor solución encontrada, lo cual es muestra de la convergencia del procedimiento de búsqueda hacia soluciones próximas a las eficientes.

Para evaluar la calidad real las soluciones finales generadas por la tarea conciliadora, se grafica el comportamiento de los indicadores de eficiencia que se obtienen en el proceso de conformación de la población final de soluciones. En las Figs. 6 y 7 se muestran el comportamiento promedio de los indicadores costo, peso y pérdidas energéticas durante la ejecución de la tarea de conciliación para cada uno de los operadores.

Como se puede observar en la Fig. 6, para el caso del OG, los indicadores de eficiencia tienden a disminuir y estabilizar su valor a un ritmo lento y escalonado. Al inicio de la búsqueda, todos los indicadores disminuyen rápidamente hasta evaluarse cerca del $7 \%$ de las soluciones, a partir de este momento se tiende a estabilizar el comportamiento de los indicadores. En el caso del indicador peso se sigue disminuyendo casi hasta el $85 \%$ de las evaluaciones, mostrando una tendencia hacia la búsqueda del mínimo de la función objetivo. El indicador costo se mantiene con pequeñas variaciones, el indicador 


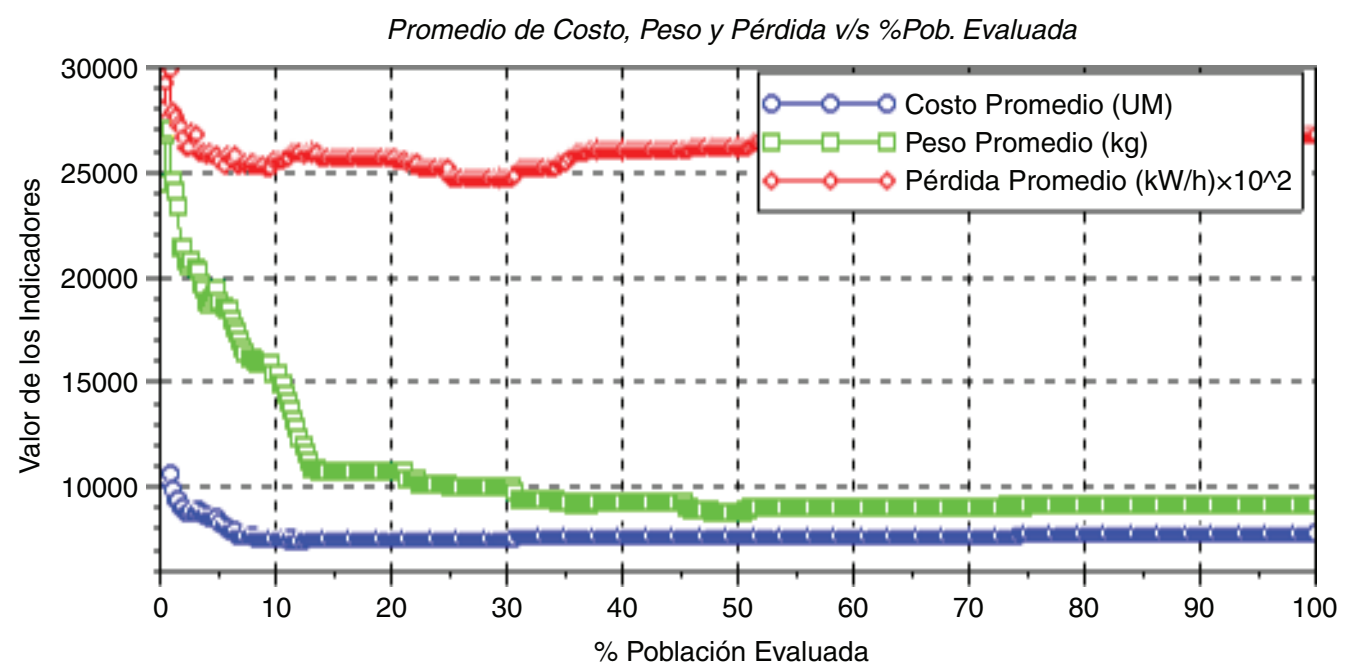

FiguRa 7. Comportamiento de los valores de los indicadores promedios de eficiencia de la población en la ejecución de la tarea conciliadora para el caso de aplicación del operador de búsqueda aleatoria (OBA).

de pérdidas energéticas tiende ligeramente a aumentar, esto se debe al propio proceso de búsqueda de soluciones no dominadas. Al tener objetivos contrapuestos, la heurística empleada sacrifica valores de otros indicadores de eficiencia en aras de mejorar la función objetivo global.

En el caso del gráfico mostrado en la Fig. 7 para el caso del OBA, se puede observar de igual modo que los indicadores al inicio de la búsqueda disminuyen rápidamente hasta cerca del $10 \%$ de las evaluaciones, en donde comienzan a variar a un ritmo más estable. Al igual que en el OG, el indicador peso disminuye a partir de este valor hasta cerca del $52 \%$ de las evaluaciones. De igual manera, el indicador pérdidas energéticas aumenta su valor hasta estabilizar por la misma razón que en el OG, lo que indica un sacrificio de su valor en la población para lograr un mejor valor global de la función multiobjetivo. Es válido resaltar que, en el caso del OBA, su comportamiento tiende a ser más estable en un porcentaje menor de evaluaciones, aunque como se puede observar en ambos gráficos, al final los dos operadores concluyen con valores muy similares para cada uno de los indicadores respectivamente.

\section{CONCLUSIONES}

- La implementación del Operador Genético inspirado en el NSGAII, bajo los principios del método de Integración de Variables, permite la creación de poblaciones de opciones de materiales y sus espesores por zona a partir de la implementación de las estrategias evolutivas de selección, reproducción y mutación empleadas junto a los principios de descomposición de la tarea planteada para la búsqueda y ordenamiento de soluciones eficientes de revestimiento basados en codificación binaria.
- La implementación en software y la experimentación realizada demuestran la validez de la utilización de códigos binarios y decimales, en correspondencia con los procedimientos propios de evolución de códigos, para el tratamiento multiobjetivo de la tarea de selección de materiales para revestimiento de cucharas de vaciado de acero.

- Las validaciones experimentales realizadas con el Operador Genético de Soluciones No Dominadas y el Operador de Búsqueda Aleatoria de Códigos Variables muestran la capacidad de ambos procedimientos para la búsqueda de soluciones de materiales y espesores según la descomposición en tareas de menor complejidad realizada a partir de la tarea original.

- La experimentación computacional realizada a partir de la implementación en ordenador de ambos operadores permite la comparación de sus desempeños. Se constata una eficiencia, para las condiciones experimentales expuestas, de un $16 \%$ superior del OBA en comparación con el OG, con un menor consumo de recursos computacionales y de tiempo, obteniéndose indicadores de eficiencia muy similares y una buena calidad en las soluciones finales de revestimiento con la aplicación de ambos procedimientos.

\section{REFERENCIAS}

Arora, S., Barak, B. (2009). Complexity theory. A modern approach. Cambridge University Press, Nueva York, pp. $40-41$.

Arzola, R.J. (2000). Sistemas de Ingeniería. Primera Edición, Editorial Félix Varela, La Habana, pp. 225-253.

Arzola, R.J. (2008). Monografía Análisis y Sintesis de Sistemas de Ingeniería. La Habana.

Arzola, R.J., Simeón, R.E., Maceo, A. (2003). El Método de Integración de Variables: una generalización de los Algoritmos Genéticos. Proc. Intensive Workshop: Optimal 
Design of Materials and Structures, Ecole Polytechnique Palaiseau, París, pp. 89-101.

Ashby, M.F. (2011). Materials Selection in Mechanical Design. Fourth Editions, Elsevier Ltd, Oxford.

Ashby, M.F., Shercliff, H., Cebon, D. (2010). Materials: Engineering, science, processing and design. Second Editions, Editorial Elsevier, Oxford.

Barbés, M.A., Martínez, E., Barbés, M.F., Alfonso, A., Verdeja, L.F. (2007). Criterios para el diseño de revestimientos que contactan con fluidos a elevadas temperaturas. FUNDIPress 2, pp. 26-30.

Bassir, D.H., Tang, X.G., Zhang, W.H. (2008). Multi-objective optimization in composites materials. EngOpt 2008, International Conference on Engineering Optimization, Rio University, Brazil.

Boisse, P., Gasser, A., Poirier, J., Rousseau, J. (2001). Simulations of thermomechanical behavior of composite refractory linings. Compos. Part B-Eng. 32 (5), 461-474. https:// dx.doi.org/10.1016/S1359-8368(01)00005-1.

Brifcani, N. (2012). A Review of Cutting-edge Techniques for Material Selection. $2^{\text {nd }}$ International Conference on Advanced Composite Materials and Technologies for Aerospace Applications, Wrexham, United Kingdom.

Chatterjee, P., Chakraborty, S. (2012). Material selection using preferential ranking methods. Mater. Design 35, 384-393. https://dx.doi.org/10.1016/j.matdes.2011.09.027.

Chawla, S.L., Gupta, R.K. (2010). Materials Selection for Corrosion Control. ASTM International, Digital printing, Materials Park, Michigan.

Chlup, Z., Boccaccini, D.N., Leonelli, C., Romagnoli, M., Boccaccini, A.R. (2006). Fracture behavior of refractory ceramics after cyclic thermal shock. Ceramics - Silikáty 50 (4), 245-250.

Clark, D.E., Folz, D.C., McGee, T.D. (2002). An introduction to Ceramic Engineering Design. Materials Selection Methodo$\log y$. Wiley-Blackwel, Ohio, USA, p. 446.

Coello, C.A., Van Veldhuizen, D.A., Lamont, G.B. (2007). Evolutionary Algorithms for Solving Multi-Objective Problems. Second Edition, Springer Science + Business Media, New York.

Deb, K., Pratap, A., Agarwal, S., Meyarivan, T. (2002). A fast and elitist multiobjective genetic algorithm: NSGA-II. IEEE T. Evolut. Comput. 6 (2), 182-197. https://dx.doi. org/10.1109/4235.996017.

DIDIER (1996). Refractory Products and Services. Didier-Werke AG, Germany.

Findik, F., Turan, K. (2012). Materials selection for lighter wagon design with a weighted property index method. Mater. Design 37, 470-477. https://dx.doi.org/10.1016/j. matdes.2012.01.016.

Hromkovič, J. (2011). Theoretical computer science: introduction to Automata, computability, complexity, algorithmics, randomization, communication, and cryptography. Springer, pp. 177-178.

Jahan, A., Ismail, M.Y., Sapuan, S.M., Mustapha, F. (2010). Material screening and choosing methods. A review. Mater. Design 31 (2), 696-705. https://dx.doi.org/10.1016/j. matdes.2009.08.013.

Jahan, A., Mustapha, F., Ismail, M.Y., Sapuan, S.M., Bahraminasab, M. (2011). A comprehensive VIKOR method for material selection. Mater. Design 32 (3), 1215-1221. https://dx.doi.org/10.1016/j.matdes.2010.10.015.

Jiménez, G.L. (2009). Optimización. Primera Edición, Sección de Publicaciones e Imagen, Manizales.

Knowles, J.D., Corne, D.W. (2000). Approximating the nondominated front using the Pareto archived evolution strategy. Evol. Comput. 8 (2), 149-172. https://dx.doi. org/10.1162/106365600568167.

Kollat, J.B., Reed, P.M. (2005). The Value of Online Adaptative Search: A Performance Comparison of NSGAII, e-NSGAII and e-MOEA. Springer-Verlag, pp. 386-398.

Lewandowski, A., Wierzbicki, A.P. (1990). Aspiration based decision support systems. Theory, software and applications. Springer-Verlag, New York.
Marrero, S., González, I., Legra, A.A. (2015). Análisis de la convergencia del método de integración de variables aplicado en la optimización multiobjetivos de la compensación de potencia reactiva en redes de suministro eléctrico. DYNA 82 (190), 160-165. http://dx.doi.org/10.15446/dyna. v82n190.43697.

Martínez, O. (2011). Optimización del Revestimiento refractario para cazuelas metalúrgicas. Tesis de Maestría, Universidad de Holguín.

Martínez, O., Arzola, R.J. (2016). Selección óptima bajo criterios múltiples de materiales refractarios y aislantes para cazuelas metalúrgicas. Rev. Int. Metod. Numer. 32 (4), 252-260. http://dx.doi.org/10.1016/j.rimni.2015. 08.001 .

Marzouk, M.M. (2011). ELECTRE III model for value engineering applications. Automat. Constr. 20 (5), 596-600. https:// dx.doi.org/10.1016/j.autcon.2010.11.026.

Méndez, M.B. (2008). Algoritmos Evolutivos y Preferencias del Decisor Aplicados a Problemas de Optimización Multiobjetivo Discretos. Tesis Doctoral, Universidad de Las Palmas de Gran Canaria, España.

Prietl, T., Zach, O., Studnicka, H. (2006). The Evaluation of Refractory Linings Thermo-Mechanical Properties. World of Metallurgy - ERZMETALL 59 (3), 127-132.

Rai, D., Kumar, G., Chatterjee, P., Chakraborty, S. (2013). Material Selection in Manufacturing Environment Using Compromise Ranking and Regret Theory-based Compromise Ranking Methods: A Comparative Study. Univer. J. Mater. Sci. 1 (2), 69-77. http://www.hrpub.org/journals/article_ info.php?aid=409.

Sakundarini, N., Thaa, Z., Hanim, S., Taha, Z., Ghazila, R., Gonzales, J. (2013). Multi-objective optimization for high recyclability material selection using genetic algorithm. Int. J. Adv. Manuf. Tech. 68 (5), 1441-1451. https://dx.doi. org/10.1007/s00170-013-4933-x.

Sapuan, S.M. (2011). A knowledge-based system for materials selection in mechanical engineering design. Mater. Design 22 (8), 687-695. https://dx.doi.org/10.1016/S02613069(00)00108-4.

Schacht, C., Maupin, M. (2004). Refractory Lining Design and Installation, in: Refractories Handbook. Schacht, C. (Ed.), Marcel Dekker Inc., New York, pp. 475-490.

Shanian, A., Savadogo, O. (2006). TOPSIS multiple-criteria decision support analysis for material selection of meta1lic bipolar plates for polymer electrolyte fuel cell. J. Power Sources 159 (2), 1095-1104. https://dx.doi.org/10.1016/j. jpowsour.2005.12.092.

Srinivas, N., Deb, K. (1994). Muiltiobjective Optimization Using Nondominated Sorting in Genetic Algorithms. J. Evol. Comput. 2 (3), 221-248. https://dx.doi.org/10.1162/ evco.1994.2.3.221.

Sukul, G., Balaramakrishna, P.V. (2011). Steady state heat transfer design considerations for refractory lining in process plants. Refractories Applications and News 16 (5), 23-32.

Toranzo, L.G., Arzola, R.J. (2014). Un algoritmo del método de Integración de Variables para la solución del problema máximo clique ponderado. Revista de Investigación Operacional 35 (1), 27-34. file:///C:/Users/02666537D/Downloads $/ \mathrm{Art} \% \mathrm{C} 3 \%$ ADculo $\% 20 \mathrm{Gustavo}$.pdf

Wegener, I. (2005). Complexity theory: exploring the limits of efficient algorithms. Springer-Verlag, New York, pp. 20.

Wei, M.L., Singh, K.N. (1986). Design of refractory lining and steel shell for caster ladles. Iron and Steel Engineer 16 (2), 61-69.

Zabolotsky, A.V. (2011). Thermal crack growth modeling in refractory linings of metallurgical installations. International Journal of Mathematical Models and Methods in Applied Sciences 5 (3), 542-548.

Zitzler, E., Thiele, L. (1999). Multiobjective evolutionary algorithms: A comparative case study and the strength Pareto approach. IEEE T. Evolut. Comput. 3 (4), 257-271. https:// dx.doi.org/10.1109/4235.797969. 